\title{
An LC-MS/MS method for multi-mycotoxin quantification in cow milk
}

\author{
Myra E. Flores-Flores, Elena González-Peñas*. \\ Organic and Pharmaceutical Chemistry Department, Faculty of Pharmacy and Nutrition, \\ University of Navarra. \\ C/ Irunlarrea 1, 31008, Pamplona, Navarra, Spain.
}

*Corresponding author: Elena González-Peñas PhD: Tel: +34 948 425653. Fax: +34

948 425652. E-mail: mgpenas@unav.es

E-mail addresses of all coauthors: Myra E. Flores-Flores: mflores.6@alumni.unav.es

\begin{abstract}
The simultaneous quantification of 15 mycotoxins in cow milk by liquid chromatography-mass spectrometry, is presented. Extraction was performed with acidified acetonitrile, followed by a cleanup step with sodium acetate. During validation limits of detection (LOD) and quantification, linearity, precision, accuracy, recovery, matrix effect, and stability were studied. LOD values were between 0.02 and 10.14 $\mathrm{ng} / \mathrm{mL}$ for aflatoxins M1, B1, B2, G1, G2, ochratoxins A and B, HT-2 and T-2 toxins, deepoxy-deoxynivalenol, zearalenone, sterigmatocystin and fumonisins B1, B2 and B3. Recovery values were between 82.6 and $94.4 \%$ for all the mycotoxins, except for fumonisins. The recovery values for fumonisins were between $42.1 \%$ and $64.6 \%$. Matrix effect, between 25.5 and $96.8 \%$, appeared for all of the mycotoxins, especially for deepoxy-deoxynivalenol, zearalenone and sterigmatocystin. The validated method achieves the quantification of those mycotoxins of major concern and mycotoxins that are not frequently studied in milk, such as fumonisins, sterigmatocystin or ochratoxin B.
\end{abstract}

\section{Keywords}

Mycotoxin, aflatoxin, fumonisin, ochratoxin, milk, LC-MS/MS.

\section{Introduction}


Agricultural products intended for human and animal consumption can be contaminated by secondary toxic fungal metabolites called mycotoxins. Contamination can occur before and/or during harvest, or when they are not properly stored (i.e. inadequate conditions of temperature and humidity) (Binder, 2007). Mycotoxins from animal feed can reach animal tissues or fluids, raw material used in the production of food for human consumption. Contaminated food and feed can cause several health problems in humans and animals, as well as economic losses, all of which has been widely reported by many authors (Capriotti, Caruso, Cavaliere, Foglia, Samperi, \& Lagana, 2012; FinkGremmels, 2008; Hussein \& Brasel, 2001; Wu, Groopman, \& Pestka, 2014). It is likely that different mycotoxins appear simultaneously in the same substrate. This is because one type of fungus can produce several mycotoxins, and frequently, an infested substrate contains diverse types of molds. In the case of co-occurrence of several mycotoxins in a single product, additive or synergic toxic effects can be expected, although they are generally unknown (Capriotti et al., 2012).

The metabolism processes in the rumen can act as a barrier against mycotoxins, and therefore, it is thought that ruminants suffer the toxic effects of mycotoxins less than other animals (Hussein \& Brasel, 2001). However, in our recent review regarding the presence of mycotoxins in milk, approximately $10 \%$ of milk samples analyzed in the world presented aflatoxin M1 (AFM1) in levels higher than the maximum level permitted in the EU. In addition, the occurrence of fumonisin B1 (FB1), fumonisin B2 (FB2), ochratoxin A (OTA), zearalenone (ZEA) and its metabolites, deepoxydeoxynivalenol (DOM-1), cyclopiazonic acid, aflatoxin G1 (AFG1), aflatoxin G2 (AFG2), aflatoxin B1 (AFB1), aflatoxin B2 (AFB2) and aflatoxin M2 (AFM2) have been detected in animal milk samples, although the levels were fairly low (FloresFlores, Lizarraga, López de Cerain, \& González-Peñas, 2015). In addition, Huang et al. detected simultaneous contamination with 2, 3 and 4 mycotoxins (AFM1, OTA, ZEA and $\alpha$-zearalenol) in $15 \%, 45 \%$ and $22 \%$ of the 50 analyzed milk samples (including liquid and powder samples), respectively (Huang et al., 2014). Moreover, certain cow diseases and/or high contamination in feed could alter the ruminal conditions and, subsequently, the rumen metabolism, thereby favoring the presence of mycotoxins in milk (Fink-Gremmels, 2008). Sørensen and Elbæk detected DOM-1 in milk samples when cows showed symptoms of illness that could not be diagnosed (Sørensen \& 
Elbæk, 2005). With regard to food processing procedures, most mycotoxins remain moderately stable (Bullerman \& Bianchini, 2007) and can remain in the food product.

The most studied mycotoxins in milk are aflatoxins and ochratoxin A, and to a lesser extent, trichothecenes and zearalenone. Others, such as ochratoxin B (OTB), sterigmatocystin (STC) and fumonisins (FB), have not been widely studied. Validated analytical methods are needed for mycotoxin determination in milk. These methods will allow studying the presence of mycotoxins in milk and the carry-over of these substances from feed to milk; they are also helpful for carrying out milk surveillance programs. Because animals are exposed to multiple mycotoxins through diet, the developed methods should be capable of multi-mycotoxin determination. These multianalyte methods allow shorter analysis times and less expensive sample surveillance, although due to their very different physicochemical properties, only the development of chromatographic techniques with mass spectrometer as the detector has allowed the simultaneous determination of several mycotoxins from different families. Moreover, the complexity of milk composition containing fat, proteins sugar and other components, make sample treatment difficult and, usually, different cleanup steps are necessary after extraction.

To the authors' knowledge, publications regarding methods especially designed for multi-mycotoxin quantification in milk are scarce (Aguilera-Luiz, Plaza-Bolaños, Romero-González, Martínez Vidal, \& Frenich, 2011; Flores-Flores \& González-Peñas, 2015; Huang et al., 2014; Sørensen \& Elbæk, 2005; Wang \& Li, 2015; Winkler, Kersten, Valenta, Meyer, Engelhardt, \& Dänicke, 2015; Zhan et al., 2012).

In this paper, a validated method capable of simultaneous determination of 15 mycotoxins (aflatoxins M1, B1, B2, G1 and G2, OTA and OTB, fumonisins B1, B2 and B3, DOM-1, T-2 and HT-2, ZEA and STC) in cow milk using liquid chromatography and mass spectrometry (triple quadrupole) is presented. This method includes the analysis of mycotoxins that have not been frequently studied in milk, such as OTB, STC and FB3. This method, with a simple extraction procedure, has been successfully applied to the analysis of 15 mycotoxins in 10 cow milk samples available in the markets throughout the region of Navarre (Spain). 


\section{Experimental section}

\subsection{Reagents}

Deionized water $\left(>18 \mathrm{M} \Omega \mathrm{cm}^{-1}\right.$ resistivity) was purified in an Ultramatic Type I system from Wasserlab (Spain). Methanol and acetonitrile (ACN) were of LC-MS and HPLC grade, respectively. In addition, formic acid (mass spectrometry grade, purity $>98 \%$ ), ammonium formate (analytical grade) and sodium acetate (anhydrous, HPLC grade $>$ 99.0\%) were used. All the reagents were purchased from Sigma-Aldrich (USA), with the exception of ACN, that was from Merck (Germany),

\subsection{Mycotoxin standard solutions}

Mycotoxins (purity $\geq 98 \%$ ) were obtained as standard solutions from Sigma-Aldrich (USA). They were stored at $-20^{\circ} \mathrm{C}$. AFM1 and OTB were at $10 \mu \mathrm{g} / \mathrm{mL}$ in ACN. AFG2 and AFB2 were at $0.5 \mu \mathrm{g} / \mathrm{mL}$ in ACN. AFG1 and AFB1 were at $2 \mu \mathrm{g} / \mathrm{mL}$ in ACN. DOM-1, T-2, HT-2, and ZEA were at $100 \mu \mathrm{g} / \mathrm{mL}$ in ACN. STC was at $50 \mu \mathrm{g} / \mathrm{mL}$ in ACN. FB1, FB2 and FB3 were at $50 \mu \mathrm{g} / \mathrm{mL}$ in $\mathrm{ACN}: \mathrm{H}_{2} \mathrm{O}$ (50:50). OTA was purchased (also from Sigma-Aldrich) in powder form and dissolved in methanol to achieve a solution of $1 \mathrm{mg} / \mathrm{mL}$. The concentration of this OTA solution was determined by UV spectrophotometry at $333 \mathrm{~nm}$ (UVIKON 922, Kontron Instruments).

Two mixed stock solutions in acetonitrile were prepared. Stock solution 1 (including all the mycotoxins, except fumonisins) was prepared by taking a defined volume from each one of the individual standard solutions and then performing adequate dilution. This solution was aliquoted ( $1 \mathrm{~mL}$ tubes $)$ and stored at $-20^{\circ} \mathrm{C}$. Prior to use, each one of these tubes was temperated in darkness for 30 min. Stock solution 2 (including only fumonisins) was freshly prepared before use. Table 1 shows the calculated concentration of each mycotoxin in the two stock solutions. Preparation of these two stock solutions was needed due to the poor stability of fumonisins in ACN. They were used for spiking milk in the preparation of calibration samples during the validation procedure (explanation provided below).

\subsection{Safety precautions}

Mycotoxins are toxic compounds and therefore, some safety precautions have been taken before handling. First, mycotoxins were handled in solution to prevent exposure to dust. In the case of OTA, the purchased powder was dissolved in methanol in the 
sealed vial with the help of a syringe. Moreover, a face shield and gloves were used when handling mycotoxin solutions and calibration samples; low-light conditions were used to prevent mycotoxin photodegradation.

\subsection{Instrumentation and analytical conditions}

The analytical instrument was a liquid chromatograph coupled to a mass spectrometer (triple quadrupole), obtained from Agilent Technologies. With regard to chromatographic conditions, a liquid chromatograph (1200 series) with degasser, binary pump, thermostated autosampler and column compartment was used. The analytical column was an Ascentis Express C18, 150 x $2.1 \mathrm{~mm}, 2.7 \mu \mathrm{m}$ particle size, fused core technology $(1.7 \mu \mathrm{m}$ solid core and a $0.5 \mu \mathrm{m}$ porous shell) from Supelco Analytical (USA). This column has been used to achieve the benefits of sub-2 $\mu \mathrm{m}$ particles (better peak resolution and less retention time) without high backpressure. The mobile phase for the chromatographic separation was a mixture of A (aqueous solution of $5 \mathrm{mM}$ ammonium formate and $0.1 \%$ formic acid) and B (aqueous-methanolic solution 5:95, containing ammonium formate, $5 \mathrm{mM}$, and formic acid, 0.1\%). Separation was performed in the following gradient conditions: $0 \mathrm{~min}, 40 \% \mathrm{~B}$, to $72 \% \mathrm{~B}$ at $20 \mathrm{~min}$, to $85 \% \mathrm{~B}$ at $20.1 \mathrm{~min}$ and maintained for $1 \mathrm{~min}$. Finally, the column was re-equilibrated for 9 min with the initial composition of the mobile phase. The thermostat on the column was set at $45^{\circ} \mathrm{C}$ during separation, and the flow of the mobile phase was $0.4 \mathrm{~mL} / \mathrm{min}$. The injection volume was $20 \mu \mathrm{L}$.

Detection and quantification of the mycotoxins were carried out using a triple quadrupole mass spectrometer (6410 Agilent Technologies) with an electrospray ionization source. Data acquisition parameters (i.e. monitoring ion pairs, fragmentor voltage, and collision energy) were optimized for each one of the mycotoxins. Quantification of mycotoxins was performed in selected reaction monitoring (SRM) mode. The mass analyzers Q1 and Q3 were operated at unit mass resolution. To prevent soiling the electrospray ionization (ESI) chamber, the effluent from the chromatographic column was diverted to waste the first $1 \mathrm{~min}$ and from min 21 until the end of the run. Other conditions were: $4000 \mathrm{~V}$ in the capillary voltage, nitrogen was used as nebulizing and drying gas at $350^{\circ} \mathrm{C}$, at $9 \mathrm{~L} / \mathrm{min}$ and $40 \mathrm{psi}$. In addition, nitrogen (99.999\% purity, Praxair, Spain) was used in the collision cell. 


\subsection{Calibration samples preparation}

Milk in which mycotoxins were not detected was spiked with the toxins from stock solutions 1 and 2. Different calibration milk samples were prepared to cover the desired concentration range for each one of the mycotoxins during the validation process. For achieving the desired concentration level, appropriate volumes of each one of the mixed stock solutions were poured into $15 \mathrm{~mL}$ centrifuge tubes, followed by reduction to approximately $25 \mu \mathrm{L}$, under vacuum and at $65^{\circ} \mathrm{C}$ (evaporator GeneVac, SP Scientific, England). The residual volume in each tube was diluted with $1 \mathrm{~mL}$ of cow milk and mixed using a vortex mixer. In this way, each one of the calibration milk samples contained all the mycotoxins simultaneously. This mixture was set aside for $10 \mathrm{~min}$ before applying the sample preparation procedure.

\subsection{Sample preparation}

One $\mathrm{mL}$ of whole cow milk was added to four milliliters of $\mathrm{ACN}(2 \% \mathrm{HCOOH})$ and this mixture was mixed using a rotary agitator during $15 \mathrm{~min}$. Next, the tube was centrifuged for $10 \mathrm{~min}$ at $5000 \mathrm{rpm}$, allowing separation of a supernatant. Four milliliters of the supernatant were separated and added to a tube with approximately 60 $\mathrm{mg}$ of sodium acetate. The tube was shaken for $10 \mathrm{~min}$ in a rotary agitator and then centrifuged for $10 \mathrm{~min}(5000 \mathrm{rpm})$. Next, $3.2 \mathrm{~mL}$ of the acetonitrile phase (upper phase) were separated and placed into another tube and evaporated at $65{ }^{\circ} \mathrm{C}$ until dryness. Finally, $200 \mu \mathrm{L}$ of $40 \% \mathrm{~B}$-mobile phase (initial composition of the mobile phase during the chromatographic separation) were added, and the tube was vortexed until the residue was dissolved (2 min). Once filtered (PVDF, $0.45 \mu \mathrm{m}$, Merck Millipore, Ireland), $20 \mu \mathrm{L}$ were injected for chromatographic analysis.

\subsection{Validation of the method}

The following parameters were studied for method validation: selectivity, detection (LOD) and quantification (LOQ) limits, linearity, precision and accuracy, recovery, matrix effect and stability.

In the analysis of the samples, the triple quadrupole mass spectrometer (QqQ) was used in SRM mode. For each one of the mycotoxins, one precursor ion and two product ions (transitions) were selected. One of the product ions (the most intense one) was chosen for quantification (Q) and the other for qualification (q); the ratio between the 
qualification and quantification transitions intensity, in percentage, was calculated in both standard samples (mycotoxins dissolved in mobile phase 40\%B) and milk calibration samples. This study was carried out for each mycotoxin, at 3 levels of concentration. The presence of the two selected transitions from the precursor ion, a mean relationship (q/Q in \%) with no more than a $15 \%$ difference between the two types of samples, and similar chromatographic retention times in standards and in milk samples $( \pm 0.5 \%)$, have demonstrated selectivity.

In order to determine LOD and LOQ for each mycotoxin, milk samples spiked at different low concentration levels, per triplicate, were prepared and processed. The LOQ for each one of the mycotoxins has been defined as the lowest concentration level for which precision and accuracy values are not higher than $20 \%$.

Precision has been calculated as RSD in percentage, whereas accuracy has been calculated as the relative error (RE), in percentage, between the nominal concentration value in the calibration sample and the concentration obtained using the calibration curve. The LOQ value has been included as the lowest level in the calibration curve for the corresponding mycotoxin. LOD was defined for each one of the mycotoxins as the concentration level in milk calibration samples in which the least intense transition ion achieved a signal-to-noise $(\mathrm{S} / \mathrm{N})$ ratio of at least three.

For the determination of range and linearity of the method, 6 milk calibration samples containing all the mycotoxins were prepared and analyzed. This procedure was repeated on three different days. Matrix-matched calibration curves were prepared for each one of the mycotoxins and the evaluation criteria for them were: determination coefficient $\left(r^{2}\right)>0.99$, slope statistically different from $0(p=95 \%)$, and accuracy, as relative error in percentage for each mycotoxin in each one of the calibration samples, $<15 \%$ except for the LOQ level (20\%).

Precision and accuracy of the method were studied in within-run (repeatability and accuracy in one day) and between-run conditions (intermediate precision and intermediate accuracy). Calibration milk samples at three concentration levels (LOQ, medium and high in the calibration range of each one of the mycotoxins) and per triplicate were prepared on the same day (within-run conditions) and on three different days (between-run conditions). The relative standard deviation and the relative error, 
both in $\%$, of the concentrations obtained for each concentration level (3 or 9 for withinrun and between-run, respectively) were given as precision and accuracy, respectively.

In addition, recovery and matrix effect were studied. These validation parameters were analyzed at three concentration levels (LOQ, medium and high levels in the range of each one of the mycotoxins) prepared per triplicate on the same day (within-run) and on three different days (between-run conditions)

Recovery (in percentage) was calculated as the ratio between the mean peak area obtained for each mycotoxin and concentration level when calibration milk samples were analyzed, and the mean peak area obtained when mycotoxins were dissolved in extracted milk. For each one of the assayed concentration levels, appropriate volumes of mixed stock solutions 1 and 2 were poured into six tubes and reduced to $25 \mu \mathrm{L}$ (approximately). Next, $1 \mathrm{~mL}$ of cow milk was added to 3 of the tubes and they were treated as described in the sample preparation section. The other 3 tubes were added with $3.2 \mathrm{~mL}$ of the acetonitrile extract obtained from a non-spiked milk sample, evaporated and resuspended in $200 \mu \mathrm{L}$ of mobile phase $(40 \% \mathrm{~B})$ before chromatographic analysis. The acceptation criteria were those established in the Commission Regulation (EC) $\mathrm{N}^{\mathrm{o}} 401 / 2006$ which established the methods for sampling and analysis for the official control of the levels of mycotoxins in foodstuffs (European Commission, 2014).

In the case of matrix effect, this was calculated as the ratio between the mean peak area obtained for each mycotoxin and concentration level when mycotoxins were dissolved in extracted milk (prepared as in the recovery study), and the mean peak area in mobile phase. In this case, the $25 \mu \mathrm{L}$ obtained after evaporating the volumes from the stock solutions were dissolved with mobile phase $(40 \% \mathrm{~B})$ until the desired concentration was obtained. The acceptance criteria were that no matrix effects appear if the ratio (in between-run conditions) was $100 \%$, and values higher or lower than $100 \%$ indicated signal enhancement or suppression, respectively.

Stability of the mycotoxins was studied in the injector compartment kept at $4^{\circ} \mathrm{C}$, in stock solution 1 at $-20^{\circ} \mathrm{C}$ and in extracted, dried and frozen, milk samples. With respect to stability in the injector, three calibration milk samples at three concentrations levels (LOQ, medium and high levels) were prepared and injected after 0, 41, 88 and $120 \mathrm{~h}$ in 
the injector compartment. These samples were analyzed along with calibration curves, and the concentration obtained for each mycotoxin was compared with the theoretical nominal value. A relative error less than $15 \%$ for each concentration level was the acceptation criterion for stability.

Stability of the mycotoxins in stock solution 1 at $-20^{\circ} \mathrm{C}$ was assessed by analyzing an aliquot of this solution stored for 19 days at $-20^{\circ} \mathrm{C}$ along with a fresh solution. Aliquots were reduced to approximately $25 \mu \mathrm{L}$ at $65^{\circ} \mathrm{C}$ and diluted in mobile phase $(40 \% \mathrm{~B})$. The stability of stock solution 2 was not studied because it was freshly prepared before use. The peak areas obtained for each mycotoxin in fresh and frozen solutions were compared, and stability was assumed if the relative error between both was less than $15 \%$.

Finally, the stability of the mycotoxins in three extracted calibration milk samples, at three concentration levels (low, medium and high levels in the calibration range of each mycotoxin) and kept at $-20^{\circ} \mathrm{C}$, was assayed. After preparing the calibration milk samples and carrying out extraction and evaporation of the ACN phase, the samples were kept at $-20^{\circ} \mathrm{C}$. This procedure was carried out for three weeks, and in the third week, all the frozen tubes were reconstituted with mobile phase and analyzed. In the evaluation for stability, a regression study (peak area-time) was carried out for each mycotoxin and concentration level. Acceptance criterion for stability was the obtainment of a slope value non-different from $0(p=95 \%)$ for each one of the three concentration levels assayed for mycotoxin.

\section{Results and discussion}

3.1 Optimization of mass spectrometry and chromatography conditions

For each mycotoxin, the precursor and product ions, the fragmentor voltage, the collision energy and retention time were studied (Table 2). With regard to MS detection, the best results were obtained when ESI was used in positive mode. The presence of $[\mathrm{M}+\mathrm{H}]^{+},\left[\mathrm{M}+\mathrm{NH}_{4}\right]^{+}$and $[\mathrm{M}+\mathrm{Na}]^{+}$adducts was studied. The most abundant adducts were chosen for almost all the mycotoxins, with the exception of AFM1. For this mycotoxin, the sodium adduct presented the highest abundance but was unable to generate reproducible product ions. Table 2 also shows the precursor and product ions chosen for mycotoxin quantification and qualification and most of them have also been 
chosen by other authors (Beltrán et al., 2013; Koesukwiwat, Sanguankaew, \& Leepipatpiboon, 2014; Mol, Plaza-Bolanos, Zomer, de Rijk, Stolker, \& Mulder, 2008; Tsiplakou, Anagnostopoulos, Liapis, Haroutounian, \& Zervas, 2014).

Different gradients and column temperatures were assayed in order to obtain chromatographic separation, and the described conditions achieve separation for all the mycotoxins in $20.1 \mathrm{~min}$. A typical chromatogram obtained during the analysis of calibration milk samples at LOQ level is shown in Fig. 1.

\subsection{Preparation of the mixed stock solutions}

To assure equivalent concentrations between different used batches of mixed stock solutions 1 and 2, an aliquot of each batch was reduced to approximately $25 \mu \mathrm{L}$, diluted with mobile phase $(40 \% \mathrm{~B})$ and analyzed. This procedure has been performed per triplicate. The mean peak areas obtained for each mycotoxin from the different batches of the mixed stock solutions were calculated and compared. The acceptance criterion for no difference among different batches was that the mean areas for each mycotoxin did not differ by more than $5 \%$.

\subsection{Procedure for milk spiking}

In order to obtain calibration samples at the desired range of concentrations, milk should be spiked with the stock solutions of mycotoxins. However, care should be taken in order to avoid altering the physicochemical properties of the milk. Two different procedures for spiking were tested. The first one consisted of drying the corresponding volume of both stock solutions ( 1 and 2 ) in a $15 \mathrm{~mL}$ polypropylene centrifuge tube and then redissolving the mycotoxins in $1 \mathrm{~mL}$ of milk by vortexing $(2 \mathrm{~min})$. The second procedure consisted of evaporating the volume of both stock solutions ( 1 and 2 ) in the same tube until they were reduced to approximately $25 \mu \mathrm{L}$. Next, this volume was mixed with $1 \mathrm{~mL}$ of milk, and $10 \mathrm{~min}$ were allowed to elapse before extraction. A better response (higher peak areas) and less RSD (\%) were obtained by applying the second method. An explanation for the failure of the first procedure could be that mycotoxins were not completely dissolved in the milk. In the second procedure, the fortification volume $(25 \mu \mathrm{L})$, representing about $2.5 \%$ of the milk volume, was small enough to cause visible disturbance of the physical characteristics of the milk. Therefore, the last procedure was chosen. 


\subsection{Extraction procedure}

Different solvents have been reported in the scientific literature for the extraction of mycotoxins from milk (Mol et al., 2008). Milk is a complex matrix and the components of milk, such as proteins, fat, sugars, etc. could give problems during the extraction of mycotoxins and/or produce matrix effects. Therefore, a cleanup step should be applied after liquid-liquid extraction.

In this study, acetonitrile, acetone, methanol, ethyl acetate and different mixtures of acetonitrile-acetone were chosen for testing their extraction capability. All of them were acidified with formic acid (2\%).

One milliliter of spiked milk was mixed with $4 \mathrm{~mL}$ of one of these solvents. Next, the mixture was centrifuged (12 min at $5000 \mathrm{rpm})$ and $4 \mathrm{~mL}$ of supernatant were transferred to a clean tube and evaporated at $65^{\circ} \mathrm{C}$. When the residue was dried, mobile phase $(40 \% \mathrm{~B})$ was added to the tube and the solution was vortexed during $2 \mathrm{~min}$, filtered and analyzed. All experiments were carried out in triplicate. The evaluation of the results followed the procedure indicated in Flores-Flores \& González-Peñas (2015). Briefly, the peak areas, obtained using each one of the solvents, were compared to those obtained with ACN in \%. Taking into account the 15 mycotoxins tested, the best results were obtained when ACN was used as extraction solvent (data not shown). Acid is needed to promote mycotoxin migration to the organic phase in the extraction step and to provide $[\mathrm{M}+\mathrm{H}]^{+}$ions to volatilization in $\operatorname{ESI}(+)$ mode. Formic acid is volatile and adequate for this function.

In addition, a cleanup step using sodium acetate was assayed after the liquid-liquid extraction process. The use of sodium acetate $(60 \mathrm{mg})$ decreased ACN solubility in water, achieving the separation of the aqueous and organic phases from the extract, and it cleaned the acetonitrile phase from matrix components, avoiding problems when treating the samples (Flores-Flores \& González-Peñas, 2015).

\subsection{Method validation}

Good results in the validation procedure have been obtained. In table 3 the range, LOD and LOQ values (the last one as the lowest concentration in the range), the determination coefficient, and the slope and intercept values of one of the prepared 
matrix-matched calibration curves for mycotoxin are shown. In each cases, $r^{2}$ is $>0.99$ and the slopes are significantly different from 0.

Low levels of mycotoxins are expected in milk. The European legislation has fixed a maximum permitted level of $0.05 \mu \mathrm{g} / \mathrm{kg}$ for AFM1 in milk. Therefore, methods for mycotoxin determination in milk should have LOD and LOQ values as low as possible. LOQ values obtained by different authors cannot often be easily compared due to the different procedures and criteria used. The LOQ value achieved in this paper for AFM1 is sufficient for its determination in milk. In the case of LOQ for the other mycotoxins, and to the best of our knowledge, they are better than the values reported by other authors who obtained LOQ values in a similar way (the lowest concentration level with satisfactory recovery and precision) and used the same type of equipment (triple quadrupole). Beltrán et al. (Beltrán et al., 2013) reported the following as the lowest validated concentration values: $0.4 \mu \mathrm{g} / \mathrm{kg}$ for AFG1, AFG2, AFB1, AFB2 and OTA; 4 $\mu \mathrm{g} / \mathrm{kg}$ for ZEA, T-2, FB1 and FB2 and $40 \mu \mathrm{g} / \mathrm{kg}$ for HT-2. AFM1 was not included in the analysis. Tsiplakou et al. (Tsiplakou et al., 2014) reported values of $10 \mu \mathrm{g} / \mathrm{kg}$ for OTA, ZEA, HT-2, T-2 and aflatoxins G1, G2, B1 and B2 and $0.05 \mu \mathrm{g} / \mathrm{kg}$ for AFM1. Xie et al. (Xie et al., 2015) were capable of quantifying 10 mycotoxins, achieving the following for the lowest validated concentration values: $0.02 \mu \mathrm{g} / \mathrm{kg}$ for AFM1; 0.2 $\mu \mathrm{g} / \mathrm{kg}$ for AFG1, AFG2, AFB1 and AFB2; $0.4 \mu \mathrm{g} / \mathrm{kg}$ for OTA; and $1 \mu \mathrm{g} / \mathrm{kg}$ for ZEA, DOM-1, FB1 and FB2. However, they used an extraction procedure that includes ultrasonic treatment, precipitation at a very low temperature $\left(-80^{\circ} \mathrm{C}\right)$ and double liquidliquid extraction followed by solid phase extraction. Jia et al. (Jia, Chu, Ling, Huang, \& Chang, 2014) achieved the quantification of fifty-eight mycotoxins in dairy products after applying a QuEChERS extraction procedure. These authors reported the detection capability $(\mathrm{CC} \beta)$ instead of LOQ, and their results ranged from $0.001 \mu \mathrm{g} / \mathrm{kg}$ for AFM1 and up to $0.54 \mu \mathrm{g} / \mathrm{kg}$ for STC, using high-resolution mass spectrometry (Orbitrap). Table 4 shows a comparison of the different methods capable of detecting mycotoxins in milk.

In addition, accuracy and precision are adequate. Precision (\% RSD) and accuracy $(\% \mathrm{RE})$ were $<15 \%$ in all the concentration levels assayed in within-run $(n=3)$ and between-run $(\mathrm{n}=9)$ conditions for all the mycotoxins (Table 5). 
Table 5 also shows the achieved recovery values (\%) and their precision (\% RSD) in between-run $(n=3)$ conditions. Mean recovery values in percentage ( $\%$ RSD) were: 82.6 (5.5), 86.9 (2.8), 85.6 (11.3), 86.6 (4.1), 88.2 (5.5), 86.0 (7.6), 87.9 (7.0), 88.1 (7.1), 90.7 (11.5), 42.1 (30.3), 94.4 (11.0), 86.4 (10.2), 86.8 (9.4), 53.8 (10.7) and 64.6 (10.9) for DOM-1, AFG2, AFM1, AFG1, AFB2, AFB1, HT-2, OTB, T-2, FB1, ZEA, OTA, STC, FB3 and FB2, respectively.

Recovery results for aflatoxins, OTA, ZEA, T-2, HT-2 and FB2 meet the performance criteria established (European Commission, 2014). Recovery value for FB1 (42.1\%) was lower than that stated in the Commission Regulation (60-120\%). No regulation has been indicated for the other mycotoxins (DOM-1, OTB, FB3 and STC), but with the exception of FB3 (53.8\%), all of them have recovery values higher than $80 \%$, and for all of them, the RSD values are less than $15 \%$. In the case of fumonisins, the low recovery values may be because they are readily soluble in water (Cole, Jarvis, \& Schweikert, 2003; IARC, 2002) and therefore, it is possible that they remain in the discarded water phase during the extraction. However, this step had to be maintained because it achieves a cleanup of the extract from matrix components that allows an adequate LOQ for AFM1 and good validation data for all mycotoxins different from fumonisins.

Matrix Effect results, evaluated at three levels of concentration in between-run conditions $(\mathrm{n}=3)$, are shown in table 5. Significant signal suppression was observed for most of them, with DOM-1, STC and ZEA being the most affected. Mean ME(\%) on different levels and days $(\mathrm{n}=9)$ were: $25.5,65.7,96.8,53.9,69.8,58.3,77.6,88.1,69.4$, 62.9, 36.1, 19.8, 77.5, 63.8, 61.5 for DOM-1, AFG2, AFM1, AFG1, AFB2, AFB1, HT2, OTB, T-2, FB1, ZEA, OTA, STC, FB3 and FB2, respectively, with RSD (\%) $\leq 15 \%$, except for DOM-1 which is $21.9 \%$. This can be explained by the fact that this mycotoxin has the lowest retention time $(1.55 \mathrm{~min})$; therefore, in the ionization source, this compound probably co-occurs with more matrix components than the other mycotoxins and the preparation of the sample has a great impact on the matrix effect for this mycotoxin. In fact, the obtained matrix effect RSD (\%) for DOM-1 in within-run conditions (data not shown) is lower than 13\% for each one of the three days. The varied $\mathrm{ME}$ values for the different mycotoxins are due to their different physicochemical properties and ionisation behavior. 
Stability studies

Compliance with the stability criteria allows concluding that all the mycotoxins were stable in the injector compartment kept at $4^{\circ} \mathrm{C}$ for at least $41 \mathrm{~h}$. However, DOM-1 and FB3 were also stable for $88 \mathrm{~h}$, and AFB1, AFB2, AFG1, AFG2, AFM1, OTB, HT-2 were stable up to $120 \mathrm{~h}$. All the mycotoxins (other than fumonisins) were stable at $-20^{\circ} \mathrm{C}$ for at least 2 weeks in extracted and evaporated samples. Fumonisins were stable for only one week. In addition, stability of the mycotoxins in mixed stock solution 1 at $20^{\circ} \mathrm{C}$ during 19 days has also been concluded.

\subsection{Applicability of proposed method}

Ten whole UHT cow milk samples from different trademarks were collected throughout the region of Navarre (Spain) and analyzed for mycotoxin contamination using the validated methodology previously described. Levels of mycotoxins higher than their respective LODs have not been encountered in the samples. Figure 1 shows an example of the chromatograms obtained when a sample and a calibration milk sample spiked at LOQ level are analyzed.

\section{Conclusions}

Milk is a highly consumed food due to the high content of nutrients for humans. It is especially consumed by children and young people, but it is also consumed at the adult age. Therefore, the presence of toxin compounds in milk should be avoided in order to protect human health. Validated analytical methods are needed for simultaneous mycotoxin determination, especially those capable of multi-detection, because they can be used to monitor the presence of different toxins in one single analysis, making the determination less expensive and less time-consuming. In this paper, an LC-MS/MS (triple quadrupole) method has been validated for achieving the simultaneous analysis of 15 mycotoxins in milk, including those of major concern for their prevalence and toxicity in raw materials, and some mycotoxins that have not been frequently studied in milk, such as fumonisins, sterigmatocystin or ochratoxin B. The proposed method, based on liquid-liquid extraction using acidified $\mathrm{ACN}$, and cleanup of the extract with sodium acetate, has yielded good results during the validation studies and has been applied to the analysis of 10 milk samples. No mycotoxins have been found, but more samples should be analyzed to evaluate their presence in this matrix. 


\section{Acknowledgements}

We wish to thank the "Programa de Investigación Universidad de Navarra" (PIUNA) for financial support. M. Flores Flores wishes to express her gratitude to the "Asociación de Amigos de la Universidad de Navarra" (ADA) for the grant funding. We also wish to thank to the reviewers for their comments and suggestions which have served to enhanced the quality of the manuscript.

\section{References}

Aguilera-Luiz, M., Plaza-Bolaños, P., Romero-González, R., Martínez Vidal, J. L., \& Frenich, A. G. (2011). Comparison of the efficiency of different extraction methods for the simultaneous determination of mycotoxins and pesticides in milk samples by ultra high-performance liquid chromatography-tandem mass spectrometry. Analytical and Bioanalytical Chemistry, 399, 2863-2875. Doi:10.1007/s00216-011$4670-7$

Beltrán, E., Ibáñez, M., Portolés, T., Ripollés, C., Sancho, J. V., Yusà, V., Marín, S., \& Hernández, F. (2013). Development of sensitive and rapid analytical methodology for food analysis of 18 mycotoxins included in a total diet study. Analytica Chimica Acta, 783, 39-48. Doi:10.1016/j.aca.2013.04.043

Binder, E. M. (2007). Managing the risk of mycotoxins in modern feed production. Animal Feed Science and Technology, 133, 149-166.

Doi:10.1016/j.anifeedsci.2006.08.008

Bullerman, L. B., \& Bianchini, A. (2007). Stability of mycotoxins during food processing. International journal of food microbiology, 119, 140-146.

Doi:10.1016/j.ijfoodmicro.2007.07.035 
Capriotti, A. L., Caruso, G., Cavaliere, C., Foglia, P., Samperi, R., \& Lagana, A. (2012). Multiclass mycotoxin analysis in food, environmental and biological matrices with chromatography/mass spectrometry. Mass spectrometry reviews, 31, 466-503. Doi:10.1002/mas.20351

Cole, R. J., Jarvis, B. B., \& Schweikert, M. A. (2003). 21 - Fumonisins, AAL Toxins, and Related Metabolites. In R. J. Cole, B. B. Jarvis \& M. A. Schweikert (Eds.), Handbook of Secondary Fungal Metabolites (pp. 561-612). San Diego: Academic Press. Doi:10.1016/B978-012179460-6/50367-9 http://www.sciencedirect.com/science/article/pii/B9780121794606503679

European Commission. (2014). Commission Regulation (EC) No 401/2006 of 23 February 2006 laying down the methods of sampling and analysis for the official control of the levels of mycotoxins in foodstuffs. Amended by Commission Regulations No 178/2010 and 519/2014. http://eur-lex.europa.eu/legalcontent/EN/TXT/?qid=1457455676474\&uri=CELEX:02006R0401-20140701 Accesed March 8th 2016.

Fink-Gremmels, J. (2008). Mycotoxins in cattle feeds and carry-over to dairy milk: a review. Food additives \& contaminants.Part A, Chemistry, analysis, control, exposure \& risk assessment, 25, 172-180. Doi:10.1080/02652030701823142

Flores-Flores, M. E., \& González-Peñas, E. (2015). Development and validation of a high performance liquid chromatographic-mass spectrometry method for the simultaneous quantification of 10 trichothecenes in ultra-high temperature processed cow milk. Journal of Chromatography A, 1419, 37-44. Doi:10.1016/j.chroma.2015.09.069 
Flores-Flores, M. E., Lizarraga, E., López de Cerain, A., \& González-Peñas, E. (2015). Presence of mycotoxins in animal milk: A review. Food Control, 53, 163-176. Doi:10.1016/j.foodcont.2015.01.020

Huang, L. C., Zheng, N., Zheng, B. Q., Wen, F., Cheng, J. B., Han, R. W., Xu, X. M., Li, S. L., \& Wang, J. Q. (2014). Simultaneous determination of aflatoxin M1, ochratoxin A, zearalenone and $\alpha$-zearalenol in milk by UHPLC-MS/MS. Food Chemistry, 146, 242-249. Doi:10.1016/j.foodchem.2013.09.047

Hussein, H. S., \& Brasel, J. M. (2001). Toxicity, metabolism, and impact of mycotoxins on humans and animals. Toxicology, 167, 101-134. Doi:10.1016/S0300$483 \mathrm{X}(01) 00471-1$

IARC. (2002). Fumonisin B1. Monographs on the Evaluation of Carcinogenic Risks to Humans. Some Traditional Herbal Medicines, Some Mycotoxins, Naphthalene and Styrene. International Agency for Research on Cancer. (pp. 301-366) http://monographs.iarc.fr/ENG/Monographs/vol82/index.php

Jia, W., Chu, X., Ling, Y., Huang, J., \& Chang, J. (2014). Multi-mycotoxin analysis in dairy products by liquid chromatography coupled to quadrupole orbitrap mass spectrometry. Journal of Chromatography A, 1345, 107-114. Doi:10.1016/j.chroma.2014.04.021

Koesukwiwat, U., Sanguankaew, K., \& Leepipatpiboon, N. (2014). Evaluation of a modified QuEChERS method for analysis of mycotoxins in rice. Food Chemistry, 153, 44-51. Doi:j.foodchem.2013.12.029 
Mol, H. G., Plaza-Bolanos, P., Zomer, P., de Rijk, T. C., Stolker, A. A., \& Mulder, P. P. (2008). Toward a generic extraction method for simultaneous determination of pesticides, mycotoxins, plant toxins, and veterinary drugs in feed and food matrixes. Analytical Chemistry, 80, 9450-9459. Doi:10.1021/ac801557f

Sørensen, L. K., \& Elbæk, T. H. (2005). Determination of mycotoxins in bovine milk by liquid chromatography tandem mass spectrometry. Journal of chromatography.B, Analytical technologies in the biomedical and life sciences, 820, 183-196. Doi:10.1016/j.jchromb.2005.03.020

Tsiplakou, E., Anagnostopoulos, C., Liapis, K., Haroutounian, S. A., \& Zervas, G. (2014). Determination of mycotoxins in feedstuffs and ruminant's milk using an easy and simple LC-MS/MS multiresidue method. Talanta, 130, 8-19. Doi:10.1016/j.talanta.2014.06.018

Wang, X., \& Li, P. (2015). Rapid screening of mycotoxins in liquid milk and milk powder by automated size-exclusion SPE-UPLC-MS/MS and quantification of matrix effects over the whole chromatographic run. Food Chemistry, 173, 897-904. Doi:10.1016/j.foodchem.2014.10.056

Winkler, J., Kersten, S., Valenta, H., Meyer, U., Engelhardt, U. H., \& Dänicke, S. (2015). Development of a multi-toxin method for investigating the carryover of zearalenone, deoxynivalenol and their metabolites into milk of dairy cows. Food Additives \& Contaminants: Part A, 32, 371-380.

Doi:10.1080/19440049.2015.1011714 
Wu, F., Groopman, J. D., \& Pestka, J. J. (2014). Public health impacts of foodborne mycotoxins. Annual review of food science and technology, 5, 351-372. Doi:10.1146/annurev-food-030713-092431

Xie, J., Peng, T., Zhu, A., He, J., Chang, Q., Hu, X., Chen, H., Fan, C., Jiang, W., Chen, M., Li, J., Ding, S., \& Jiang, H. (2015). Multi-residue analysis of veterinary drugs, pesticides and mycotoxins in dairy products by liquid chromatography-tandem mass spectrometry using low-temperature cleanup and solid phase extraction. Journal of Chromatography B, 1002, 19-29. Doi:10.1016/j.jchromb.2015.08.005

Zhan, J., Yu, X., Zhong, Y., Zhang, Z., Cui, X., Peng, J., Feng, R., Liu, X., \& Zhu, Y. (2012). Generic and rapid determination of veterinary drug residues and other contaminants in raw milk by ultra performance liquid chromatography-tandem mass spectrometry. Journal of Chromatography B, 906, 48-57.

Doi:10.1016/j.jchromb.2012.08.018 


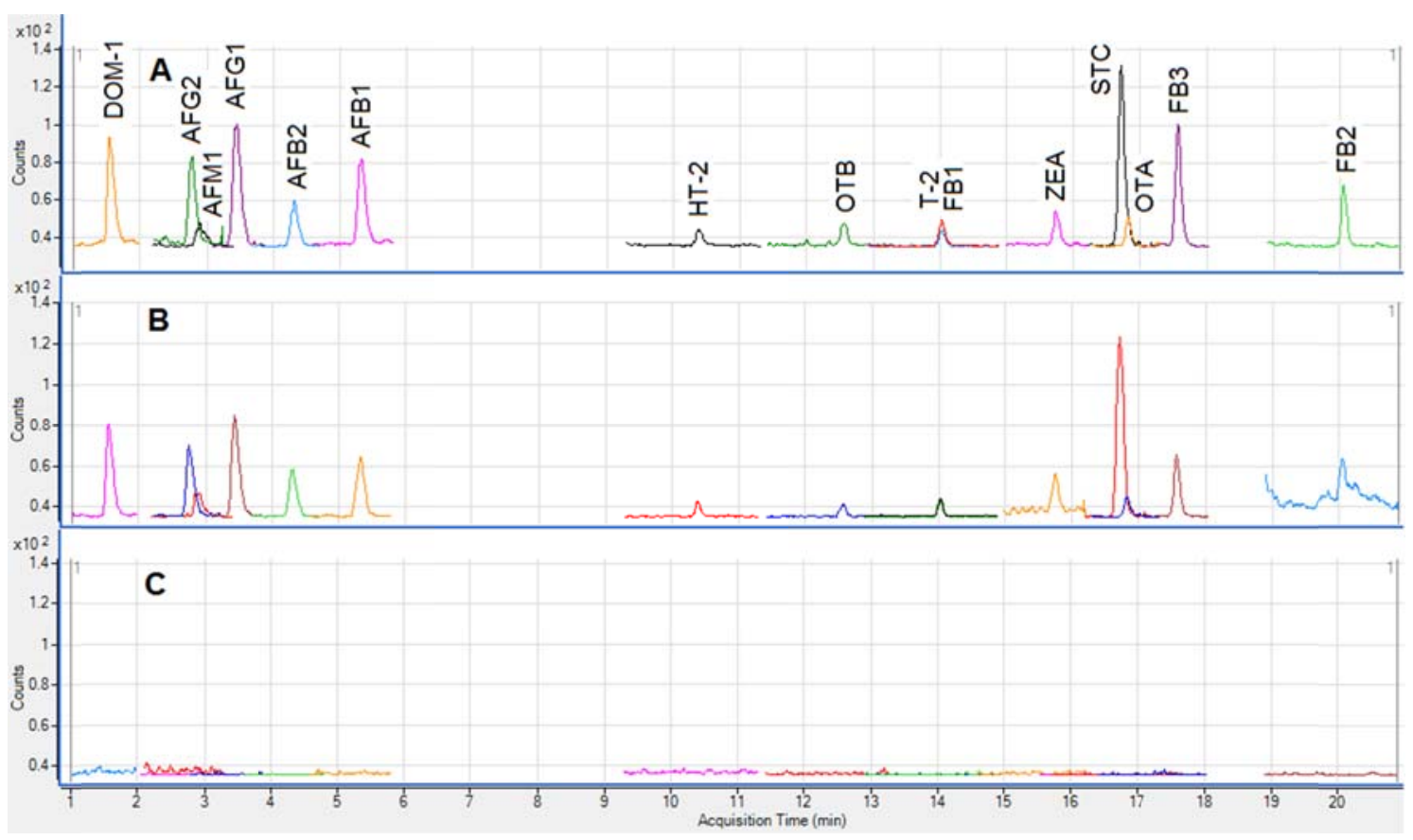

\section{Figure caption:}

Fig. 1: Superposed typical extracted chromatograms for each mycotoxin after extraction of a spiked milk sample at LOQ level (A and B) and a real milk sample (C). (A) and (C) display the quantification transition and (B) the qualification transition for each mycotoxin. 
Table 1: Mycotoxin concentrations in the mixed stock solutions 1 and 2.

\begin{tabular}{cccc}
\hline Mycotoxin & ng $\mathbf{~ m L}^{-\mathbf{1}}$ & Mycotoxin & ${\text { ng } \mathbf{~ m L}^{-1}}^{-1}$ \\
\hline \multicolumn{4}{c}{ Stock solution 1 } \\
\hline DOM-1 & 151.5 & HT-2 & 20.1 \\
AFG2 & 7.5 & OTB & 2.5 \\
AFM1 & 2.5 & T-2 & 2.5 \\
AFG1 & 5.1 & ZEA & 25.5 \\
AFB2 & 2.0 & STC & 25.1 \\
AFB1 & 2.0 & OTA & 5.1 \\
\hline \multicolumn{4}{c}{ Stock solution 2 } \\
\hline FB1 & 253.5 & FB2 & 125.0 \\
FB3 & 125.0 & \\
\hline
\end{tabular}


Table 2: Retention times and MS parameters for identifying each mycotoxin.

\begin{tabular}{|c|c|c|c|c|c|c|c|c|c|}
\hline$t_{R}{ }^{a}(\min )$ & Mycotoxin & Adduct & $\begin{array}{l}\text { Precursor } \\
\text { Ion }\end{array}$ & $\begin{array}{c}\text { Product } \\
\text { Ion } \\
(\mathbf{Q})^{\mathrm{a}} \\
\end{array}$ & $\begin{array}{l}\text { Product } \\
\operatorname{Ion}(q)^{a}\end{array}$ & $\begin{array}{c}\text { Frag. }^{a} \\
\text { (V) }\end{array}$ & $\mathrm{CE}^{\mathrm{a}}(\mathrm{V})$ & $\begin{array}{c}\text { Relative } \\
\text { Intensity } \\
\text { \%(sample) }\end{array}$ & $\begin{array}{c}\text { Relative } \\
\text { Intensity } \\
\text { \%(standard) }\end{array}$ \\
\hline 1.55 & DOM-1 & $\mathrm{H}^{+}$ & 281.2 & 233.1 & 215.2 & 90 & $5 / 5^{b}$ & 91 & 92 \\
\hline 2.77 & AFG2 & $\mathrm{H}^{+}$ & 331.1 & 313.1 & 285.1 & 160 & $20 / 30$ & 78 & 78 \\
\hline 2.91 & AFM1 & $\mathrm{H}^{+}$ & 329.0 & 273.0 & 229.1 & 140 & $20 / 50$ & 101 & 95 \\
\hline 3.45 & AFG1 & $\mathrm{H}^{+}$ & 329.0 & 243.1 & 199.0 & 150 & $26 / 57$ & 69 & 70 \\
\hline 4.33 & AFB2 & $\mathrm{H}^{+}$ & 315.0 & 259.0 & 287.1 & 160 & $30 / 23$ & 95 & 91 \\
\hline 5.33 & AFB1 & $\mathrm{H}^{+}$ & 313.0 & 128.1 & 285.1 & 160 & $83 / 20$ & 59 & 58 \\
\hline 10.41 & HT2 & $\mathrm{NH}_{4}^{+}$ & 442.2 & 215.1 & 197.1 & 90 & $7 / 10$ & 55 & 54 \\
\hline 12.56 & ОТВ & $\mathrm{H}^{+}$ & 370.1 & 205.2 & 187.1 & 100 & $20 / 35$ & 40 & 40 \\
\hline 14.04 & FB1 & $\mathrm{H}^{+}$ & 722.4 & 352.1 & 334.0 & 180 & $50 / 50$ & 71 & 74 \\
\hline 14.08 & $\mathrm{~T} 2$ & $\mathrm{NH}_{4}^{+}$ & 484.2 & 215.1 & 305.1 & 100 & $15 / 7$ & 57 & 61 \\
\hline 15.76 & ZEA & $\mathrm{H}^{+}$ & 319.2 & 187.1 & 283.2 & 100 & $20 / 5$ & 99 & 98 \\
\hline 16.74 & STC & $\mathrm{H}^{+}$ & 325.0 & 310.1 & 281.0 & 150 & $25 / 35$ & 86 & 87 \\
\hline 16.87 & OTA & $\mathrm{H}^{+}$ & 404.1 & 239.0 & 102.1 & 120 & $25 / 80$ & 71 & 72 \\
\hline 17.61 & FB3 & $\mathrm{H}^{+}$ & 706.4 & 336.5 & 95.1 & 190 & $37 / 50$ & 75 & 75 \\
\hline 20.08 & FB2 & $\mathrm{H}^{+}$ & 706.3 & 336.2 & 54.9 & 180 & $40 / 70$ & 46 & 45 \\
\hline
\end{tabular}

${ }^{a} t_{R}$ : retention time. Q: quantification. q: qualification. Frag: Fragmentor. CE: Collision energy.

${ }^{b t}$ the first value corresponds to $(\mathrm{Q})$ and second value to $(\mathrm{q})$.

${ }^{c}$ Relative intensity calculated as $\mathrm{q} / \mathrm{Q} * 100$, mean of values obtained at three concentrations. 
Table 3: Linear range, LOD and regression data for a typical calibration curve

\begin{tabular}{lcccccc}
\hline Mycotoxin & $\begin{array}{c}\text { Range } \\
(\mathrm{ng} / \mathrm{mL})\end{array}$ & $\begin{array}{c}\text { LOD } \\
(\mathrm{ng} / \mathrm{mL})\end{array}$ & $\mathrm{r}^{2}$ & \multicolumn{3}{c}{ slope } \\
(confidence interval 95\%) & intercept \\
\hline DOM-1 & $3.03-30.30$ & 0.758 & 0.9993 & 117.15 & $(114.55-119.74)$ & 7.49 \\
AFG2 & $0.15-1.50$ & 0.075 & 0.9986 & 2334.85 & $(2263.61-2406.1)$ & 17.76 \\
AFM1 & $0.05-0.50$ & 0.025 & 0.9986 & 1659.81 & $(1587.4-1732.21)$ & 24.54 \\
AFG1 & $0.10-1.02$ & 0.025 & 0.9992 & 5173.61 & $(5075.63-5271.58)$ & -8.42 \\
AFB2 & $0.04-0.40$ & 0.020 & 0.9997 & 4477.49 & $(4424.55-4530.44)$ & 10.87 \\
AFB1 & $0.04-0.40$ & 0.020 & 0.9998 & 8991.84 & $(8886.66-9097.03)$ & 0.97 \\
HT2 & $0.40-4.02$ & 0.400 & 0.9982 & 166.66 & $(161.64-171.68)$ & 4.48 \\
OTB & $0.05-0.50$ & 0.050 & 0.9988 & 1612.66 & $(1555.97-1669.36)$ & 7.97 \\
T2 & $0.05-0.50$ & 0.050 & 0.9991 & 943.89 & $(918.87-968.9)$ & 1.99 \\
FB1 & $10.14-50.70$ & 10.140 & 0.9985 & 7.54 & $(7.2-7.87)$ & 9.96 \\
ZEA & $0.51-5.09$ & 0.510 & 0.9977 & 216.48 & $(208.06-224.89)$ & 7.67 \\
STC & $0.50-5.02$ & 0.125 & 0.9982 & 1139.77 & $(1105.28-1174.26)$ & 11.04 \\
OTA & $0.20-1.00$ & 0.200 & 0.9958 & 428.00 & $(400.25-455.76)$ & -2.27 \\
FB3 & $2.50-25.00$ & 0.625 & 0.9985 & 155.69 & $(151.71-159.67)$ & 14.54 \\
FB2 & $2.50-25.00$ & 2.500 & 0.9992 & 64.44 & $(63.09-65.79)$ & 26.99 \\
\hline
\end{tabular}


1 Table 4: Comparison of the different published methods able to detect mycotoxins in milk.

\begin{tabular}{|c|c|c|c|c|c|c|c|c|c|c|c|c|c|c|c|c|c|c|}
\hline \multirow[t]{2}{*}{ Reference } & \multicolumn{15}{|c|}{ Validated Limit of Quantification ( $\mu \mathrm{g} / \mathrm{kg}$ ) } & \multirow[t]{2}{*}{ Detector } & \multirow[t]{2}{*}{ Matrix } & \multirow[t]{2}{*}{ Sample treatment } \\
\hline & AFM1 & AFG2 & AFG1 & AFB2 & AFB1 & OTA & ZEA & HT-2 & T-2 & FB1 & FB2 & FB3 & STC & OTB & DOM-1 & & & \\
\hline Zhan et al. 2012 & 4 & 4 & 4 & 4 & 4 & 4 & 4 & & & & & & & & & QQQ & milk & Double LLE \\
\hline Tsiplakou et al. 2014 & 0.05 & 10 & 10 & 10 & 10 & 10 & 10 & 10 & 10 & & & & & & & QQQ & feed and milk & LLE, freezing \\
\hline Beltrán et al. 2013 & & 0.4 & 0.4 & 0.4 & 0.4 & 0.4 & 4 & 40 & 4 & 4 & 4 & & & & & QQQ & food & LLE \\
\hline Wang and Li 2015 & 0.01 & & & & 0.01 & 0.01 & 0.01 & & & & & & & & & QQQ & milk & Automated SPE \\
\hline Sørensen and Elbæk 2005 & 0.02 & & & & & & & 0.2 & 0.2 & 0.2 & 0.2 & & & & & QQQ & milk & LLE and SPE \\
\hline Sørensen and Elbæk 2005 & & & & & & 0.05 & 0.05 & & & & & & & & 0.2 & QQQ & milk & LLE and SPE \\
\hline Xie et al. 2015 & 0.02 & 0.2 & 0.2 & 0.2 & 0.2 & 0.4 & 1 & & & 1 & 1 & & & & 1 & QQQ & dairy products & $\begin{array}{c}\text { Double LLE, freezing } \\
\text { and SPE }\end{array}$ \\
\hline Huang et al. 2014 & 0.025 & & & & & 0.025 & 0.025 & & & & & & & & & QQQ & milk & LLE and SPE \\
\hline Aguilera-Luiz et al. 2011 & 0.05 & 0.5 & 0.5 & 0.5 & 0.5 & & & & 0.5 & & & & & & & QQQ & milk & SPE \\
\hline Jia et al. 2014 & 0.001 & 0.007 & 0.005 & 0.009 & 0.009 & 0.009 & 0.08 & 0.09 & & 0.08 & 0.09 & 0.54 & 0.02 & 0.01 & 0.03 & Orbitrap & dairy products & $\begin{array}{l}\text { LLE, cleanup (salts) } \\
\text { and SPE }\end{array}$ \\
\hline Winkler et al. 2015 & & & & & & & 80 & & & & & & & & 160 & Qtrap & milk & SPE \\
\hline Proposed method & 0.05 & 0.15 & 0.1 & 0.04 & 0.04 & 0.2 & 0.51 & 0.4 & 0.05 & 10.14 & 2.5 & 2.5 & 0.5 & 0.05 & 3 & QQQ & milk & LLE and cleanup (sal \\
\hline
\end{tabular}

2 LLE: liquid-liquid extraction, SPE: solid phase extraction, QQQ: triple quadrupole 
5 Table 5: Precision (\%RSD), accuracy (\%RE), matrix effect (\%ME) and recovery (\%R). Within-run precision and accuracy have been studied in triplicate at

6 each concentration level, whereas between-run have been studied in triplicate on three different days. ME and recovery have been studied in between-run

7 conditions on three days.

\begin{tabular}{|c|c|c|c|c|c|c|c|c|c|c|c|c|c|c|c|c|c|c|c|c|c|c|c|c|}
\hline \multirow[t]{3}{*}{ Mycotoxin } & \multicolumn{6}{|c|}{ Precision (RSD) \% } & \multicolumn{6}{|c|}{ Accuracy (RE) \% } & \multirow{2}{*}{\multicolumn{6}{|c|}{$\begin{array}{l}\text { Matrix effect \% (RSD\%) } \\
\text { Between-run (n=3) }\end{array}$}} & \multirow{2}{*}{\multicolumn{6}{|c|}{$\begin{array}{c}\text { Recovery \% }\left(\mathrm{RSD}_{0}\right) \\
\text { Between-run }(\mathrm{n}=3)\end{array}$}} \\
\hline & \multicolumn{3}{|c|}{ Within-run ( $n=3$ ) } & \multicolumn{3}{|c|}{ Between-run ( $\mathrm{n}=9$ ) } & \multicolumn{3}{|c|}{ Within-run $(n=3)$} & \multicolumn{3}{|c|}{ Between-run $(n=9)$} & & & & & & & & & & & & \\
\hline & $\mathrm{L}^{*}$ & $\mathrm{M}$ & $\mathrm{H}$ & $\mathrm{L}$ & $\mathrm{M}$ & $\mathrm{H}$ & $\mathrm{L}$ & $\mathrm{M}$ & $\mathrm{H}$ & $\mathrm{L}$ & $\mathrm{M}$ & $\mathrm{H}$ & $\mathrm{L}$ & & $\mathrm{M}$ & & $\mathrm{H}$ & & $\mathrm{L}$ & & $\mathrm{M}$ & & $\mathrm{H}$ & \\
\hline DOM-1 & 7.9 & 6.3 & 0.8 & 6.0 & 5.8 & 1.2 & 0.1 & 0.2 & 0.3 & 2.8 & 0.5 & 0.3 & 26.1 & (22.9) & 23.3 & (24.9) & 27.2 & $(21.8)$ & 81.0 & $(4.9)$ & 82.5 & $(4.0)$ & 84.3 & $(8.2)$ \\
\hline AFG2 & 7.6 & 6.5 & 3.1 & 9.4 & 6.8 & 3.9 & 2.0 & 0.9 & 0.9 & 2.6 & 2.4 & 0.2 & 68.9 & $(11.8)$ & 61.9 & (10.6) & 66.2 & (13.6) & 85.9 & $(3.0)$ & 87.8 & (3.9) & 87.1 & $(1.8)$ \\
\hline AFM1 & 13.5 & 6.5 & 7.1 & 9.6 & 5.0 & 6.5 & 0.2 & 1.9 & 0.7 & 5.3 & 0.0 & 1.8 & 110.5 & (11.4) & 90.1 & $(6.6)$ & 89.8 & $(6.8)$ & 93.7 & (3.4) & 83.1 & $(8.5)$ & 80.1 & $(15.9)$ \\
\hline AFG1 & 4.4 & 7.3 & 2.2 & 6.9 & 6.7 & 3.2 & 1.5 & 2.1 & 0.9 & 3.4 & 0.5 & 0.3 & 56.1 & $(6.1)$ & 51.5 & (9.3) & 54.1 & (12.6) & 84.9 & (5.6) & 85.0 & $(2.3)$ & 89.9 & $(2.2)$ \\
\hline AFB2 & 10.3 & 8.8 & 0.9 & 10.8 & 8.2 & 4.1 & 6.2 & 3.0 & 1.1 & 1 & 1.6 & 0.2 & 69.3 & (7.6) & 69.5 & (8.3) & 70.7 & $(11.2)$ & 89.4 & (4.6) & 87.0 & $(9.8)$ & 88.3 & (1.6) \\
\hline AFB1 & 2.0 & 6.9 & 0.8 & 5.3 & 6.4 & 3.4 & 3.4 & 0.7 & 0.0 & 4.4 & 0.9 & 0.3 & 58.6 & $(7.3)$ & 57.5 & $(7.0)$ & 58.8 & (13.9) & 88.5 & (6.1) & 79.9 & (10.1) & 89.6 & (3.3) \\
\hline HT-2 & 7.7 & 8.6 & 4.2 & 10.6 & 7.3 & 5.3 & 2.1 & 2.6 & 0.8 & 1.4 & 1.3 & 0.3 & 81.9 & (3.9) & 73.4 & (7.1) & 77.7 & $(6.0)$ & 86.4 & (7.4) & 90.1 & $(8.2)$ & 87.3 & (7.7) \\
\hline ОТВ & 8.3 & 12.7 & 4.3 & 9.3 & 8.4 & 6.6 & 3.3 & 0.3 & 0.1 & 1.3 & 1.3 & 0.2 & 88.3 & $(3.5)$ & 84.2 & (3.4) & 91.7 & $(3.7)$ & 88.6 & $(8.2)$ & 88.6 & $(9.0)$ & 87.1 & (9.3) \\
\hline $\mathrm{T}-2$ & 12.1 & 7.7 & 8.0 & 9.5 & 6.4 & 7.3 & 1.2 & 7.1 & 1.7 & 0.9 & 2.6 & 0.8 & 70.9 & (9.8) & 63.7 & $(14.3)$ & 73.5 & (11.3) & 96.0 & (10.7) & 93.4 & (14.3) & 82.7 & (2.3) \\
\hline FB1 & 9.1 & 5.5 & 9.1 & 7.1 & 7.6 & 5.5 & 2.7 & 2.8 & 0.9 & 2.1 & 3.0 & 0.1 & 59.9 & (15.8) & 61.1 & (3.3) & 67.6 & (8.8) & 58.8 & (7.1) & 34.7 & (8.4) & 32.6 & $(5.0)$ \\
\hline ZEA & 9.8 & 5.4 & 12.1 & 11.2 & 6.3 & 7.9 & 4.2 & 1.4 & 0.4 & 5.1 & 2.1 & 0.9 & 35.9 & (4.6) & 34.4 & (10.7) & 38.0 & $(8.2)$ & 101.5 & $(12.2)$ & 94.4 & (17.7) & 87.2 & (20.9) \\
\hline STC & 4.1 & 8.4 & 7.8 & 10.3 & 8.9 & 5.8 & 3.7 & 5.2 & 2.6 & 7.8 & 2.3 & 2.5 & 20.4 & $(4.6)$ & 18.9 & (12.7) & 20.2 & (11.4) & 89.9 & (2.4) & 88.8 & (9.6) & 81.7 & $(14.0)$ \\
\hline OTA & 13.4 & 6.1 & 5.7 & 10.8 & 8.5 & 5.7 & 0.5 & 3.3 & 1.6 & 1.3 & 0.5 & 0.6 & 72.6 & (11.9) & 77.4 & $(4.3)$ & 82.4 & (10.6) & 84.7 & (12.6) & 87.4 & $(8.8)$ & 87.0 & (13.1) \\
\hline FB3 & 5.9 & 6.2 & 3.3 & 10.4 & 6.4 & 4.1 & 10.4 & 0.4 & 0.9 & 0.2 & 2.3 & 0.4 & 58.6 & (13.7) & 59.6 & (6.1) & 66.3 & $(6.5)$ & 54.1 & (9.4) & 51.3 & (11.9) & 56.1 & $(13.0)$ \\
\hline FB2 & 4.6 & 8.3 & 3.8 & 6.9 & 6.4 & 5.0 & 2.0 & 0.1 & 0.1 & 5.5 & 2.3 & 2.6 & 62.1 & (12.7) & 60.0 & $(0.9)$ & 69.2 & $(2.1)$ & 66.9 & $(5.2)$ & 58.1 & $(13.0)$ & 68.8 & $(10.2)$ \\
\hline
\end{tabular}

$9 * \mathrm{~L}, \mathrm{M}, \mathrm{H}$ : low, medium and high concentration levels of each mycotoxin, respectively. 